\title{
Tool Demonstration of the FLATA Counter Automata Toolset*
}

\author{
Marius Bozga ${ }^{1}$, Radu Iosif ${ }^{1}$, Filip Konečný ${ }^{2}$ and Tomáš Vojnar $^{2}$ \\ 1 VERIMAG, CNRS \\ Gières, France \\ 2 Brno University of Technology \\ Brno, Czech Republic
}

\begin{abstract}
FLATA is a toolset for the manipulation and the analysis of non-deterministic integer programs (also known as counter automata). The input to the tool is a textual description of a counter automaton. The main functionalities of FLATA are:

- checking emptiness of the model, i.e. deciding if a final state is reachable from an initial state

- reducing the size of the input model to smaller models with equivalent emptiness problems

- exporting to other counter automata formats, such as Fast and ARMC

In this talk we demonstrate the capabilities of the tool for checking reachability in counter automata using techniques which have recently been developed (such as precise acceleration of self-loops labelled by DBMs or octagons) and/or which are still under development. Given that reachability problems in such models are undecidable, in cases where the analysis cannot be completed, we can still generate a reduced model with the same relation between the input and output counter values, which can be fed to another tool. For demonstrating the tool, we use counter automata obtained, e.g., by translation from list manipulating programs or hardware components.
\end{abstract}

${ }^{*}$ This work was supported by the French national project ANR-09-SEGI-016 VERIDYC, by the Czech Science Foundation (projects P103/10/0306 and 102/09/H042), the Czech Ministry of Education (projects COST OC10009 and MSM 0021630528), and the internal FIT BUT grant FIT-S-10-1. 